\title{
Un cas de métastase gingivale d'un carcinome hépato-cellulaire
}

\author{
Nicolas Davido ${ }^{1}$, Jonathan Mimran ${ }^{1}$, Elina Teicher ${ }^{3}$, Françoise Gruffaz ${ }^{2}$, Rafaël Toledo ${ }^{1}$ \\ ${ }^{1}$ Groupe hospitalier Pitié-Salpêtrière, Service d'Odontologie, 47-83 boulevard de l'Hôpital, 75013 \\ Paris, France \\ ${ }^{2}$ Groupe hospitalier Pitié-Salpêtrière, Service d'Anatomie et de Cytologie pathologiques, 47-83 \\ boulevard de l'Hôpital, 75013 Paris, France \\ ${ }^{3}$ Hôpital Kremlin Bicêtre, Service de Médecine interne, 78, rue du Général Leclerc, 94275 Le \\ Kremlin-Bicêtre Cedex, France
}

nicolas.davido@gmail.com

Le carcinome hépato-cellulaire (CHC) est la deuxième cause de cancer digestif en France. Il représente 0,5 à $2 \%$ de l'ensemble des cancers avec environ 6000 nouveaux cas par an en France. Le CHC survient sur foie cirrhotique dans plus de $85 \%$ des cas, principalement d'origine alcoolique ou virale (Blanc 2007). La plupart des patients sont des hommes (ratio homme-femme, $46: 4$ ) de plus de 50 ans dans $90 \%$ des cas (Chin et al. 1998). Les métastases extra-hépatiques surviennent dans 25 à $37 \%$ des cas (Ramirez et al. 2003 ; Fujihara et al. 2010) et touchent préférentiellement les poumons, les ganglions lymphatiques régionaux, le système musculo-squelettique, les glandes surrénales et rarement la région buccale (Katyal et al. 2000 ; Pires et al. 2004). Les métastases buccales de CHC sont présentes dans $30 \%$ des cas avant la découverte du cancer primitif (Kuo et al. 2006). C'est alors 1'os mandibulaire qui est le plus souvent atteint (Vigneul et al. 1982, Li et al. 2008). Les métastases gingivales sont rarrissimes (Morishita et Fukuda 1984, Maiorano et al. 2000, Pires et al. 2004, Kuo et al. 2006). Il n'y a que 15 cas de métastase gingivale du CHC rapportés (Kuo et al. 2006). Lorsque celle-ci est présente, elle est souvent associée à d'autres localisations, notamment pulmonaires (Maiorano et al. 2000).

Le cas présenté est un nouveau cas de métastase gingivale d'un $\mathrm{CHC}$ au niveau du maxillaire droit survenant chez un homme caucasien de 50 ans co-infecté VIH-VHC, au stade cirrhose. Le patient est adressé par l'hôpital Paul Brousse aux urgences odontologiques du Groupe hospitalier Pitié-Salpêtrière pour un saignement de la cavité buccale depuis 3 jours. Les antécédents dentaires rapportent la notion d'une avulsion dentaire par le patient lui-même dans les jours qui précèdent l'apparition du saignement buccal.

A l'examen, il existe une tuméfaction d'aspect inflammatoire, modérément hémorragique, hémisphérique plutôt bien limitée, mesurant $2 \mathrm{~cm}$, siégeant sur la crête maxillaire droite édentée après la 14, entourant une racine résiduelle de la16, faisant évoquer cliniquement un granulome pyogénique. L'examen radiologique retrouve cette tuméfaction sans atteinte osseuse ni extension sinusienne droite.

On a réalisé en urgence l'avulsion de la racine résiduelle de la 16 et l'exérèse de la tuméfaction. L'examen anatomo-pathologique a mis en évidence une prolifération carcinomateuse dont l'aspect histologique et immunohistochimique étaient évocateurs d'un carcinome d'aspect hépatocellulaire et devaient faire recherche une tumeur hépatique primitive. Cette tumeur a été confirmée dans le service où le patient était hospitalisé. La métastase gingivale est la seule localisation extra-hépatique du CHC et le patient est décédé 16 semaines après la découverte de son CHC. 